\title{
On Deformations and Contractions of Lie Algebras
}

\author{
Alice FIALOWSKI ${ }^{\dagger}$ and Marc DE MONTIGNY ${ }^{\ddagger}$ \\ † Institute of Mathematics, Eötvös Loránd University, Pázmány Péter sétány 1/C, \\ H-1117, Budapest, Hungary \\ E-mail: fialowsk@cs.elte.hu \\ URL: http://www.cs.elte.hu/ fialowsk/ \\ $\ddagger$ Campus Saint-Jean and Theoretical Physics Institute, University of Alberta, 8406 - 91 Street, \\ Edmonton, Alberta, T6C 4G9, Canada \\ E-mail: montigny@phys.ualberta.ca \\ URL: http://www.phys.ualberta.ca/〜montigny/
}

Received February 24, 2006, in final form April 25, 2006; Published online May 03, 2006

Original article is available at http://www.emis.de/journals/SIGMA/2006/Paper048/

\begin{abstract}
In this contributed presentation, we discuss and compare the mutually opposite procedures of deformations and contractions of Lie algebras. We suggest that with appropriate combinations of both procedures one may construct new Lie algebras. We first discuss low-dimensional Lie algebras and illustrate thereby that whereas for every contraction there exists a reverse deformation, the converse is not true in general. Also we note that some Lie algebras belonging to parameterized families are singled out by the irreversibility of deformations and contractions. After reminding that global deformations of the Witt, Virasoro, and affine Kac-Moody algebras allow one to retrieve Lie algebras of Krichever-Novikov type, we contract the latter to find new infinite dimensional Lie algebras.
\end{abstract}

Key words: Lie algebras; deformations; contractions; Kac-Moody algebras

2000 Mathematics Subject Classification: 17B66; 17B67; 17B65; 17B56; 17B68; 14D15; $81 \mathrm{~T} 40$

\section{Introduction}

The purpose of this presentation is to report on a recent analysis of two different approaches to 'deformations' of Lie algebras, by which loose term we mean continuous modifications of their structure constants. These deformations appear in mathematics and in physics under various guises. We analyze hereafter two main categories of such modifications: contractions, which typically transform a Lie algebra into a 'more Abelian' Lie algebra, and deformations, which, understood in a strict sense, lead to Lie algebras with more intricate Lie brackets. There exists a plethora of definitions for both contractions and deformations and below, we recall general definitions within the framework of Lie algebras. In order to clarify the two concepts and provide explicit constructions, we consider concrete examples in low dimensions and infinite dimensions. For further details and references, see [1].

References about early works on deformations of mathematical structures in general, and Lie algebras in particular, are given in [1. We have listed some of them in [2]. Of particular interest hereafter is the more general deformation theory obtained by considering an arbitrary commutative algebra with unity as the base of deformation. Such deformations are called 'global' and appear in the work of Fialowski and Schlichenmaier [3].

A contraction is a procedure somewhat opposite to deformation. Contractions are important in physics because they explain in terms of Lie algebras why some theories arise as a limit regime of more 'exact' theories. They consist in multiplying the generators of the symmetry 
by 'contraction parameters' such that when these parameters reach some singularity point one obtains a non-isomorphic Lie algebra with the same dimension [4]. The mathematics literature contains various concepts similar to contractions: 'degeneration', 'orbit closure', etc.

Now let us define both concepts. A review of the concepts of deformations and contractions is given in [5]. Some articles which address various aspects of both deformations and contractions are in [6]. Consider a Lie algebra $\mathfrak{g}$ of dimension $N$ over the field $k$ which we take hereafter as being $k=\mathbb{R}$ and $\mathbb{C}$. We write the basis elements of $\mathfrak{g}$ as $\left\{x_{1}, \ldots, x_{N}\right\}$ with Lie brackets

$$
\left[x_{i}, x_{j}\right]=C_{i j}^{k} x_{k},
$$

where the coefficients $C_{i j}^{k}$ are the structure constants. We denote by $\mathcal{L}_{N}(k)$ the space of structural tensors of $N$-dimensional Lie algebras. A one-parameter deformation of a Lie algebra $\mathfrak{g}$, with structure constants belonging to $\mathcal{L}_{N}(k)$, can be seen as a continuous curve over $\mathcal{L}_{N}(k)$. One refers to the deformation as being (piecewise) smooth, analytic, etc. if the associated defining curve itself is (piecewise) smooth, analytic, respectively.

A formal one-parameter deformation is defined by the Lie brackets:

$$
[a, b]_{t}=F_{0}(a, b)+t F_{1}(a, b)+\cdots+t^{m} F_{m}(a, b)+\cdots,
$$

where $F_{0}$ denotes the original Lie bracket $[\cdot, \cdot]$ and $F_{m}$ are two-cochains. The Jacobi identity implies, among others, that $F_{1}$ must be a two-cocycle of $\mathfrak{g}$. We call $[\cdot, \cdot]_{t}$ a first-order, or infinitesimal, deformation if it satisfies the Jacobi identity up to $t^{2}$. It follows that first-order deformations correspond to elements of the space of two-cocycles $Z^{2}(\mathfrak{g}, \mathfrak{g})$. When a formal deformation is such that $[,]_{t} \simeq[,]_{s}$ for every $t$ and $s$ except 0 , then we call it a jump deformation. It is clear from the definition that contractions (defined in equation (3)) are exactly related to jump deformations.

Now let us look at a deformation $\mathfrak{g}_{t}=[\cdot, \cdot]_{t}$ not as a one-parameter family of Lie algebras, but as a Lie algebra over the ring $k[[t]]$ of formal power series over $k$. A natural generalization is to allow more parameters, which amounts to consider $k\left[\left[t_{1}, \ldots, t_{k}\right]\right]$ as the base, or, even more generally, to take an arbitrary commutative algebra $A$ over $k$, with unit as the base. Assume that $A$ admits an augmentation $\epsilon: A \rightarrow k$, such that $\epsilon$ is a $k$-algebra homomorphism and $\epsilon\left(1_{A}\right)=1$. The ideal $m_{\epsilon}:=\operatorname{ker}(\epsilon)$ is a maximal ideal of $A$, and, given a maximal ideal $m$ of $A$ with $A / m \cong k$, the natural quotient map defines an augmentation. If $A$ has a unique maximal ideal, the deformation with base $A$ is called local. If $A$ is the projective limit of local algebras, the deformation is called formal. In Section 3, we will consider infinite dimensional Lie algebras obtained by using the concept of global deformation. For more details, see [1.

Intuitively, rigidity of a Lie algebra $\mathfrak{g}$ means that we cannot deform it. We call a Lie algebra infinitesimally rigid if every infinitesimal deformation is equivalent to the trivial one, and formally rigid if every formal deformation is trivial. The examples discussed below are formally rigid (see 9, 10]). As mentioned in Section 3, the interesting feature of infinite dimensional Lie algebras is that formal deformations are no longer sufficient to describe general deformations. The examples discussed below are formally rigid (see [9]), so that they admit no non-trivial formal deformations. Nevertheless, there exist very interesting non-trivial global deformations. In global deformation theory, we no longer have the tool of computing cohomology in order to get deformations so that the picture is much more difficult and there are very few results so far [3].

The commutation relations of a contracted Lie algebra, or contraction, $\mathfrak{g}^{\prime}$ of a Lie algebra $\mathfrak{g}$, are given by the limit [4, 7, 8,

$$
[x, y]^{\prime} \equiv \lim _{\varepsilon \rightarrow \varepsilon_{0}} \mathcal{U}_{\varepsilon}^{-1}\left(\left[\mathcal{U}_{\varepsilon}(x), \mathcal{U}_{\varepsilon}(y)\right]\right.
$$


Table 1. Three-dimensional complex Lie algebras.

\begin{tabular}{|ll|}
\hline & {$\left[x_{i}, x_{j}\right]=0, \quad i, j=1,2,3$} \\
$\mathbb{C}^{3}:$ & {$\left[x_{1}, x_{2}\right]=x_{3}$} \\
$\mathfrak{n}_{3}(\mathbb{C}):$ & {$\left[x_{1}, x_{2}\right]=x_{2}$} \\
$\mathfrak{r}_{2}(\mathbb{C}) \oplus \mathbb{C}:$ & {$\left[x_{1}, x_{2}\right]=x_{2},\left[x_{1}, x_{3}\right]=x_{2}+x_{3}$} \\
$\mathfrak{r}_{3}(\mathbb{C}):$ & {$\left[x_{1}, x_{2}\right]=x_{2},\left[x_{1}, x_{3}\right]=\lambda x_{3}$} \\
$\mathfrak{r}_{3, \lambda}(\mathbb{C}),\left(\lambda \in \mathbb{C}^{*},|\lambda| \leq 1\right):\left[x_{1}, x_{2}\right]=x_{3},\left[x_{2}, x_{3}\right]=x_{1},\left[x_{3}, x_{1}\right]=x_{2}$ \\
$\mathfrak{s l}_{2}(\mathbb{C}):$ & \\
& \\
\hline
\end{tabular}

where $\mathcal{U}_{\varepsilon} \in \operatorname{GL}(N, k)$ is a non-singular linear transformation of $\mathfrak{g}$, with $\varepsilon_{0}$ being a singularity point of its inverse $\mathcal{U}_{\varepsilon}^{-1}$.

Throughout the paper, however, we shall utilize contractions defined with diagonal $\mathcal{U}_{\varepsilon}$ in equation (3), defined by splitting the Lie algebra $\mathfrak{g}$ into an arbitrary number of subspaces:

$$
\mathfrak{g}=\mathfrak{g}_{0}+\mathfrak{g}_{1}+\cdots+\mathfrak{g}_{p},
$$

and by taking the matrix $\mathcal{U}_{\varepsilon}$ as follows:

$$
\mathcal{U}_{\varepsilon}^{\text {diag }}=\oplus_{j} \varepsilon^{n_{j}} \operatorname{id}_{\mathfrak{g}_{j}}, \quad \varepsilon>0, \quad n_{j} \in \mathbb{R}, \quad j=1,2, \ldots, p,
$$

where $p \leq \operatorname{dim} \mathfrak{g}$. From equations (10) and (5), and if we denote by $\mathfrak{g}_{i}$ the subspace in (44) to which the element $x_{i}$ belongs, then equation (3) becomes

$$
\left[x_{i}, x_{j}\right]^{\prime}=\lim _{\varepsilon \rightarrow 0} \varepsilon^{n_{i}+n_{j}-n_{k}} C_{i j}^{k} x_{k} .
$$

Thus the exponents in equation (5) must satisfy

$$
n_{i}+n_{j}-n_{k} \geq 0,
$$

unless $C_{i j}^{k}=0$. Then the structure constants of the contracted algebra $\mathfrak{g}^{\prime}$ are given by $\left(C^{\prime}\right)_{i j}^{k}=$ $C_{i j}^{k}$ if $n_{i}+n_{j}=n_{k}$, and $\left(C^{\prime}\right)_{i j}^{k}=0$ if $n_{i}+n_{j}>n_{k}$. Two trivial contractions are always present: the Abelian Lie algebra and the original Lie algebra itself, for which the commutation relations are unchanged. Likewise, an Abelian Lie algebra can be deformed to every Lie algebra of the same dimension.

\section{Three-dimensional complex Lie algebras}

In this section, we enumerate the deformations and the contractions of complex three-dimensional Lie algebras, in order to demonstrate the differences between the two concepts. Deformation of complex three-dimensional Lie algebras were recently classified in [11. The real algebras are discussed in [1].

The Lie brackets of the three-dimensional complex Lie algebras are given in Table 1 Note that $\mathfrak{r}_{3, \bar{\lambda}}(\mathbb{C})$ is isomorphic to $\mathfrak{r}_{3, \lambda}(\mathbb{C})$ when $|\lambda|=1$ because $\lambda \bar{\lambda}=1$. As a simple illustration of the methods, consider the contraction from $\mathfrak{s l}_{2}(\mathbb{C})$ to $\mathfrak{r}_{3,-1}(\mathbb{C})$. We express the Lie brackets of $\mathfrak{s l}_{2}(\mathbb{C})$ in the Cartan basis:

$$
[h, e]=e, \quad[h, f]=-f, \quad[e, f]=2 h .
$$

Then, we may introduce the contraction parameters as follows:

$$
e \rightarrow \varepsilon e, \quad f \rightarrow \varepsilon f, \quad h \rightarrow h,
$$




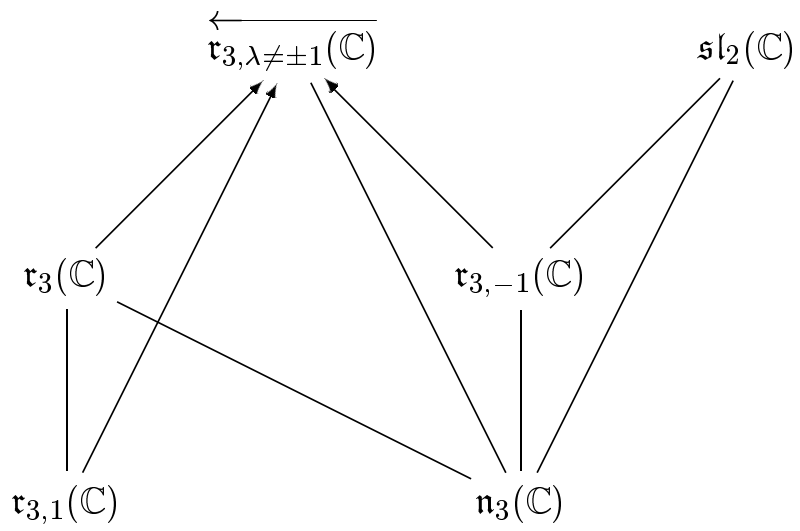

Figure 1. Contractions and deformations of the three-dimensional complex Lie algebras.

before taking the limit $\varepsilon \rightarrow 0$. This results in $[e, f] \rightarrow 0$, with $[h, e]$ and $[h, f]$ unchanged, i.e. the Lie brackets for $\mathfrak{r}_{3,-1}(\mathbb{C})$. Now, let us illustrate the reverse deformation with this simple example. The original Lie brackets of $\mathfrak{r}_{3,-1}(\mathbb{C})$ are such that, in equation (2), the non-zero $F_{0}$ 's are $F_{0}(h, e)=e$ and $F_{0}(h, f)=-f$. Then, in order to deform it to $\mathfrak{s l}_{2}(\mathbb{C})$, we may write equation (2) as

$$
[h, e]_{t}=e+t F_{1}(h, e), \quad[h, f]_{t}=-f+t F_{1}(h, f), \quad[e, f]_{t}=t F_{1}(e, f),
$$

where

$$
F_{1}(h, e)=0, \quad F_{1}(h, f)=0, \quad F_{1}(e, f)=2 h,
$$

as suggested clearly by the contraction. The resulting Lie algebra is isomorphic to $\mathfrak{s l}_{2}(\mathbb{C})$, for any non-zero $t$.

The results of contractions and deformations of three-dimensional complex Lie algebras are displayed on Fig. 1 The lines and arrows should be interpreted as follows: an arrow points toward the deformation, whereas a simple line connects Lie algebras related by both deformation and contraction, with the deformed Lie algebra lying upward. The left-pointing arrow symbol over $\mathfrak{r}_{3, \lambda \neq \pm 1}(\mathbb{C})$ means that it deforms inside the family.

Let us note once again that a non-trivial contraction always induces a non-trivial (inverse) jump deformation. The converse is not always true: there are deformations which do not admit an inverse contraction. For example, one can never have a contraction inside a parameterized family of Lie algebras, but deformations within a family are allowed. Note also that nothing can be contracted to the parameterized family, whereas there are many non-trivial deformations in dimension three to the family $\mathfrak{r}_{3, \lambda \neq \pm 1}(\mathbb{C})$. We should emphasize that the irreversibility occurs only when we have a family of smooth deformations.

The family of Lie algebras $\mathfrak{r}_{3, \lambda \neq \pm 1}(\mathbb{C})$ has a non-trivial deformation into itself. The two Lie algebras $\mathfrak{r}_{3,1}(\mathbb{C})$ and $\mathfrak{r}_{3,-1}(\mathbb{C})$ are special for two reasons. First, $\mathfrak{r}_{3,1}(\mathbb{C})$ can be deformed into $\mathfrak{r}_{3}(\mathbb{C})$, whereas $\mathfrak{r}_{3, \lambda \neq 1}(\mathbb{C})$ cannot. Moreover $\mathfrak{r}_{3,-1}(\mathbb{C})$ can deform into $\mathfrak{s l}_{2}(\mathbb{C})$, whereas $\mathfrak{r}_{3, \lambda \neq-1}(\mathbb{C})$ cannot. Second, $\mathfrak{r}_{3,1}(\mathbb{C})$ is special because it cannot be contracted to $\mathfrak{n}_{3}(\mathbb{C})$, unlike $\mathfrak{r}_{3, \lambda \neq 1}(\mathbb{C})$.

\section{Infinite dimensional Lie algebras}

The physical interest of infinite dimensional Lie algebras stems mainly from conformal field theory and critical phenomena in two dimensions [12. Whereas the Witt and Virasoro algebras describe local invariance of conformal field theories on the (zero genus) Riemann sphere, 
hereafter we shall discuss some Lie algebras of Krichever-Novikov type which correspond to higher genus. As mentioned previously, these Lie algebras are formally rigid, yet they can be deformed. These deformations are so-called global and cohomology theory then does not lend itself to compute such deformations [1. These contractions turn out to be especially useful since they often lead to new infinite-dimensional Lie algebras, as we will demonstrate now.

\subsection{Witt algebras and Krichever-Novikov algebras}

First, let us consider the Witt algebra $\mathfrak{W}$ with Lie brackets:

$$
\left[l_{n}, l_{m}\right]=(m-n) l_{n+m}, \quad n, m \in \mathbb{Z} .
$$

The deformation pattern of its only one-dimensional central extension, the Virasoro algebra, is quite similar and we will not discuss it hereafter. Krichever and Novikov introduced new algebras in 13. An interesting aspect of infinite-dimensional Lie algebras, which does not occur for the finite-dimensional cases, is that Lie algebras of Krichever-Novikov type $\mathfrak{K N}$ can be interpreted as global deformations of the Witt or Virasoro algebra [3], even though the Witt algebra $\mathfrak{W}$ is formally rigid, thus preventing any non-trivial formal deformation.

An example of Krichever-Novikov algebras that can be obtained as a one-parameter global deformation of Witt algebra is generated with the following field basis:

$$
V_{2 n} \equiv X(X-\alpha)^{n}(X+\alpha)^{n} \frac{d}{d X}, \quad V_{2 n+1} \equiv(X-\alpha)^{n+1}(X+\alpha)^{n+1} \frac{d}{d X},
$$

which satisfy the following Lie brackets:

$$
\left[V_{n}, V_{m}\right]= \begin{cases}(m-n) V_{n+m}, & n, m \text { odd } \\ (m-n)\left(V_{n+m}+\alpha^{2} V_{n+m-2}\right), & n, m \text { even } \\ (m-n) V_{n+m}+(m-n-1) \alpha^{2} V_{n+m-2}, & n \text { odd, } m \text { even. }\end{cases}
$$

Clearly this can be contracted back to the Witt algebra by defining $\mathcal{U}_{\varepsilon}$ in equations (4) and (15) as

$$
l_{n} \equiv \varepsilon^{n} V_{n}, \quad \text { for all } n \in \mathbb{Z} .
$$

Then equation (9) becomes

$$
\left[l_{n}, l_{m}\right]_{\varepsilon}=\varepsilon^{n+m}\left[V_{n}, V_{m}\right]= \begin{cases}(m-n) l_{n+m}, & n, m \text { odd } \\ (m-n)\left(l_{n+m}+\varepsilon^{2} \alpha^{2} l_{n+m-2}\right), & n, m \text { even } \\ (m-n) l_{n+m}+(m-n-1) \varepsilon^{2} \alpha^{2} l_{n+m-2}, & n \text { odd, } m \text { even }\end{cases}
$$

We retrieve the commutation relations, equation (8), of $\mathfrak{W}$ as $\varepsilon$ approaches zero. Therefore, the operations of deformation and contraction are mutually reversible in this case.

In addition to retrieving the Witt algebra $\mathfrak{W}$, equation (9), one may contract $\mathfrak{K N}$ to new Lie algebras. For instance, let us define $\mathcal{U}_{\varepsilon}$ as

$$
\mathcal{U}_{\varepsilon} \equiv \varepsilon^{n_{0}} \mathrm{id}_{\mathfrak{g}_{0}}+\varepsilon^{n_{1}} \operatorname{id}_{\mathfrak{g}_{1}},
$$

where 0 and 1 denote the even and odd powers in $\mathfrak{K N}$, respectively. This choice is quite natural, given the odd versus even splitting in equation (9). Then the Lie brackets (9) are modified to

$$
\left[V_{n}, V_{m}\right]_{\varepsilon}= \begin{cases}\varepsilon^{2 n_{1}-n_{0}}(m-n) V_{n+m}, & n, m \text { odd }, \\ \varepsilon^{n_{0}}(m-n)\left(V_{n+m}+\alpha^{2} V_{n+m-2}\right), & n, m \text { even, } \\ \varepsilon^{n_{0}}\left[(m-n) V_{n+m}+(m-n-1) \alpha^{2} V_{n+m-2}\right], & n \text { odd, } m \text { even }\end{cases}
$$


Clearly we must have non-negative values of $n_{0}$ and $2 n_{1}-n_{0}$. This leads to four separate contracted Lie algebras: (1) we obtain the trivial Abelian Lie algebra when these expressions take on positive values; (2) another trivial contraction is given by $n_{0}=n_{1}=0$; then it leaves the commutators of equation (9) unchanged; (3) the Inönü-Wigner contraction, given by $n_{0}=0$ and $n_{1}=1$, so that the contracted commutation relations read:

$$
\left[V_{n}, V_{m}\right]= \begin{cases}0, & n, m \text { odd }, \\ (m-n)\left(V_{n+m}+\alpha^{2} V_{n+m-2}\right), & n, m \text { even, } \\ (m-n) V_{n+m}+(m-n-1) \alpha^{2} V_{n+m-2}, & n \text { odd, } m \text { even }\end{cases}
$$

and (4) by choosing $n_{0}>0$ and $2 n_{1}-n_{0}=0$ in equation (12), we find

$$
\left[V_{n}, V_{m}\right]= \begin{cases}(m-n) V_{n+m}, & n, m \text { odd, } \\ 0, & n, m \text { even, } \\ 0, & n \text { odd }, m \text { even. }\end{cases}
$$

The cases (3) and (4) are clearly not isomorphic to the Witt algebra, equation (8).

\subsection{Affine Kac-Moody and Krichever-Novikov algebras}

Now let us discuss deformations and contractions of $\mathfrak{K N}$-type Kac-Moody algebras. Untwisted Kac-Moody algebras $\hat{\mathfrak{g}}=\left(\mathfrak{g} \otimes \mathbb{C}\left[t, t^{-1}\right]\right) \oplus \mathbb{C} c$ are defined in terms of a finite simple complex Lie algebra $\mathfrak{g}$ together with $\mathbb{C}\left[t, t^{-1}\right]$, the associative algebra of the Laurent polynomials, and the central extension $c$. The Lie brackets may be written as

$$
\left[a \otimes t^{m}, b \otimes t^{n}\right]=[a, b] \otimes t^{m+n}+m c B(a, b) \delta_{m+n, 0},
$$

where [, ] denotes the Lie brackets of the finite Lie algebra $\mathfrak{g}$. In [14, Majumdar showed that for Inönü-Wigner contractions, affinization and contraction are commuting procedures.

Hereafter we discuss contractions of Krichever-Novikov algebras, the latter having been obtained by global deformations of affine Kac-Moody algebras. In [3], it is shown that the trivially extended affine algebras, equation (13) with $k=0$, may be deformed to the following $\mathfrak{K N}$ type algebra, parameterized over the affine plane $\mathbb{C}^{2}$, or, described algebraically, over the polynomial algebra $\mathbb{C}\left[e_{1}, e_{2}\right]$ :

$$
\left[a \otimes A^{n}, b \otimes A^{m}\right]= \begin{cases}{[a, b] \otimes A^{n+m},} & n \text { or } m \text { even }, \\ {[a, b] \otimes A^{n+m}+3 e_{1}[a, b] \otimes A^{n+m-2}} & \\ \quad+\left(e_{1}-e_{2}\right)\left(2 e_{1}+e_{2}\right)[a, b] \otimes A^{n+m-4}, & n \text { and } m \text { odd }\end{cases}
$$

where $a$ and $b$ belong to a finite dimensional complex Lie algebra $\mathfrak{g}$. The choice $\left(e_{1}, e_{2}\right)=(0,0)$ simply leads to the original affine algebra (although this is not a contraction process).

As we have done in the previous section, let us first see that equation (14) may be contracted back to the original Kac-Moody algebra by defining the transformation $\mathcal{U}_{\varepsilon}$ in analogy with equation (10):

$$
a \otimes t^{n} \equiv \varepsilon^{n} a \otimes A^{n}, \quad \text { for all } n \in \mathbb{Z},
$$

so that the Lie brackets (14) become

$$
\left[a \otimes A^{n}, b \otimes A^{m}\right]_{\varepsilon}= \begin{cases}{[a, b] \otimes A^{n+m},} & n \text { or } m \text { even }, \\ {[a, b] \otimes A^{n+m}+3 e_{1} \varepsilon^{2}[a, b] \otimes A^{n+m-2}} & \\ \quad+\left(e_{1}-e_{2}\right)\left(2 e_{1}+e_{2}\right) \varepsilon^{4}[a, b] \otimes A^{n+m-4}, & n \text { and } m \text { odd } .\end{cases}
$$

This leads to equation (13), with $k=0$, in the limit $\varepsilon \rightarrow 0$. 
Now, let us obtain other Lie algebras by using contraction procedures similar to what we have done for the Witt algebra. Consider, for instance, the splitting of $\mathfrak{K N}$ as in equation (14) as we have done in equation (11). We find

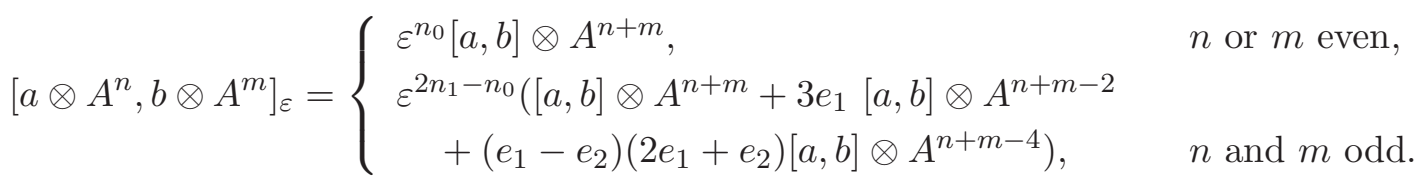

The Inönü-Wigner contraction discussed after equation (12), for which $n_{0}=0$ and $n_{1}=1$, leads to commutation relations where the first line in equation (16), i.e. for $n$ or $m$ even, remains unchanged, whereas the other commutators, given in line 2 of the same equation, vanish. If, instead, we take $n_{0}$ positive and $2 n_{1}-n_{0}=0$, then equation (16) leads to the opposite situation: the Lie brackets in the first line, i.e. for $n$ or $m$ even, will vanish in the limit $\varepsilon \rightarrow 0$, whereas the remaining commutators, given in line 2 of equation (16) are left unchanged. Evidently, there are countless possibilities, if we replace equation (15) with a different splitting of the Lie algebras.

Let us now turn to contractions where the splitting is not done only with respect to the degrees of the Laurent polynomials, but within the underlying finite Lie algebra $\mathfrak{g}$. For the sake of illustration, let us consider a $\mathfrak{K N}$ algebra based upon the Lie algebra $\mathfrak{s l}_{2}(\mathbb{C})$, and split it according to the following $\mathbb{Z}_{3}$-graded structure, inherent to the basis of equation (7):

$$
\mathfrak{s l}_{2}(\mathbb{C})=\mathfrak{s l}_{2}(\mathbb{C})_{0}+\mathfrak{s l}_{2}(\mathbb{C})_{1}+\mathfrak{s l}_{2}(\mathbb{C})_{-1}=\{h\}+\{e\}+\{f\} .
$$

We may combine this $\mathbb{Z}_{3}$-grading with the $\mathbb{Z}_{2}$-grading provided by the even versus odd degrees of the Laurent polynomials to obtain the following $\mathbb{Z}_{3} \otimes \mathbb{Z}_{2}$ grading:

$$
\begin{aligned}
\mathfrak{s l}_{2}(\mathbb{C})= & \overbrace{\left\{h \otimes A^{2 n}\right\}}^{\mathfrak{s l}_{2}(\mathbb{C})_{00}}+\overbrace{\left\{e \otimes A^{2 n}\right\}}^{\mathfrak{s l}_{2}(\mathbb{C})_{10}}+\overbrace{\left\{f \otimes A^{2 n}\right\}}^{\mathfrak{s l}_{2}(\mathbb{C})_{-10}}+\overbrace{\left\{h \otimes A^{2 n+1}\right\}}^{\mathfrak{s l}_{2}(\mathbb{C})_{01}} \\
& +\overbrace{\left\{e \otimes A^{2 n+1}\right\}}^{\mathfrak{s l}_{2}(\mathbb{C})_{11}}+\overbrace{\left\{f \otimes A^{2 n+1}\right\}}^{\mathfrak{s l}_{2}(\mathbb{C})_{-11}} .
\end{aligned}
$$

The grading property implies that

$$
\left[\mathfrak{g}_{\mu}, \mathfrak{g}_{\nu}\right]_{\varepsilon}=\varepsilon^{n_{\mu}+n_{\nu}-n_{\mu+\nu}} \mathfrak{g}_{\mu+\nu}
$$

where $\mu=a b$ is a double index with $a=\{0,1,-1\} \in \mathbb{Z}_{3}$ and $b=\{0,1\} \in \mathbb{Z}_{2}$. As in equation (6), the six exponents $n_{00}, n_{10}, n_{-10}, n_{01}, n_{11}, n_{-11}$ must satisfy $n_{\mu}+n_{\nu}-n_{\mu+\nu} \geq 0$.

The commutation relations read explicitly as

$$
\begin{aligned}
& {\left[h \otimes A^{2 n}, e \otimes A^{2 m}\right]_{\varepsilon}=\varepsilon^{n_{00}} e \otimes A^{2 n+2 m},} \\
& {\left[h \otimes A^{2 n}, e \otimes A^{2 m+1}\right]_{\varepsilon}=\varepsilon^{n_{00}} e \otimes A^{2 n+2 m+1},} \\
& {\left[h \otimes A^{2 n+1}, e \otimes A^{2 m}\right]_{\varepsilon}=\varepsilon^{n_{01}+n_{10}-n_{11}} e \otimes A^{2 n+2 m+1},} \\
& {\left[h \otimes A^{2 n+1}, e \otimes A^{2 m+1}\right]_{\varepsilon}=\varepsilon^{n_{01}+n_{11}-n_{10}}\left(e \otimes A^{2 n+2 m+2}+3 e_{1} e \otimes A^{2 n+2 m}\right.} \\
& \left.+\left(e_{1}-e_{2}\right)\left(2 e_{1}+e_{2}\right) e \otimes A^{2 n+2 m-2}\right) ; \\
& {\left[h \otimes A^{2 n}, f \otimes A^{2 m}\right]_{\varepsilon}=-\varepsilon^{n_{00}} f \otimes A^{2 n+2 m},} \\
& {\left[h \otimes A^{2 n}, f \otimes A^{2 m+1}\right]_{\varepsilon}=-\varepsilon^{n_{00}} f \otimes A^{2 n+2 m+1},} \\
& {\left[h \otimes A^{2 n+1}, f \otimes A^{2 m}\right]_{\varepsilon}=-\varepsilon^{n_{01}+n_{-10}-n_{-11}} f \otimes A^{2 n+2 m+1},} \\
& {\left[h \otimes A^{2 n+1}, f \otimes A^{2 m+1}\right]_{\varepsilon}=-\varepsilon^{n_{01}+n_{-11}-n_{-10}}\left(f \otimes A^{2 n+2 m+2}+3 e_{1} f \otimes A^{2 n+2 m}\right.} \\
& \left.+\left(e_{1}-e_{2}\right)\left(2 e_{1}+e_{2}\right) f \otimes A^{2 n+2 m-2}\right) \text {; }
\end{aligned}
$$




$$
\begin{aligned}
& {\left[e \otimes A^{2 n}, f \otimes A^{2 m}\right]_{\varepsilon}=2 \varepsilon^{n_{10}+n_{-10}-n_{00}} h \otimes A^{2 n+2 m},} \\
& {\left[e \otimes A^{2 n}, f \otimes A^{2 m+1}\right]_{\varepsilon}=2 \varepsilon^{n_{10}+n_{-11}-n_{01}} h \otimes A^{2 n+2 m+1},} \\
& {\left[e \otimes A^{2 n+1}, f \otimes A^{2 m}\right]_{\varepsilon}=2 \varepsilon^{n_{11}+n_{-10}-n_{01}} h \otimes A^{2 n+2 m+1},} \\
& {\left[e \otimes A^{2 n+1}, f \otimes A^{2 m+1}\right]_{\varepsilon}=2 \varepsilon^{n_{11}+n_{-11}-n_{00}}\left(h \otimes A^{2 n+2 m+2}+3 e_{1} h \otimes A^{2 n+2 m}\right.} \\
& \left.+\left(e_{1}-e_{2}\right)\left(2 e_{1}+e_{2}\right) h \otimes A^{2 n+2 m-2}\right) .
\end{aligned}
$$

We just illustrate a few original algebraic objects that can be obtained by contractions. It is not our purpose to find all the solutions for the $n$ 's. The first two lines of equations (17) and (18) imply that

$$
n_{00} \geq 0 .
$$

If we take $n_{00}=0$ and $n_{01}=0$, then lines 3 and 4 of equation (17) imply that $n_{11}=n_{10}$, whereas lines 3 and 4 of equation (18) lead to $n_{-10}=n_{-11}$. From the factors in equation (19), we find $n_{10}+n_{-10} \geq 0$. All these result in the fact that the commutators in equations (17) and (18) all remain unchanged, whereas the Lie brackets in equation (19) either all remain unchanged after the contraction, or they all vanish. Note that the latter contracted algebra can also be obtained by first contracting $\mathfrak{s l}_{2}(\mathbb{C})$ to $\mathfrak{r}_{3,-1}(\mathbb{C})$ and then affinizing it $\grave{a}$ la Krichever-Novikov. In other words, it follows from the Krichever-Novikov construction that the $\mathfrak{K N}$ algebra obtained directly from $\mathfrak{r}_{3,-1}(\mathbb{C})$ is the same as is we construct the $\mathfrak{K N}_{\mathfrak{N}}$ of $\mathfrak{s l}_{2}(\mathbb{C})$, and then form its contraction, in a way analogous to the contraction from $\mathfrak{s l}_{2}(\mathbb{C})$ to $\mathfrak{r}_{3,-1}(\mathbb{C})$.

Clearly, more complicated contracted algebras, where the commutators involving different powers contract differently, can be obtained. Consider, once again, the case $n_{00}=0$, but $n_{01}>0$. Then, the exponents $n_{01}+n_{10}-n_{11}, n_{01}+n_{11}-n_{10}, n_{01}+n_{-10}-n_{-11}$ or $n_{01}+n_{-11}-n_{-10}$ cannot be all equal to zero simultaneously. Consider the case where these exponents are all strictly positive. This means that lines 1 and 2 of equations (17) and (18) remain unchanged under these contractions, whereas lines 3 and 4 of the same equations will vanish in the limit $\varepsilon \rightarrow 0$. We may choose to preserve lines 1 and 4 of equation (19) by taking $n_{10}+n_{-10}=0$ and $n_{11}+n_{-11}=0$, respectively. For the sake of illustration, let us choose

$$
n_{00}=0, \quad n_{01}=n_{10}=n_{11}=1, \quad n_{-10}=n_{-11},
$$

which satisfy all these conditions. Then equations (17), (18) and (19) read

$$
\begin{aligned}
& {\left[h \otimes A^{2 n}, e \otimes A^{2 m}\right]_{\varepsilon}=\varepsilon^{0} e \otimes A^{2 n+2 m},} \\
& {\left[h \otimes A^{2 n}, e \otimes A^{2 m+1}\right]_{\varepsilon}=\varepsilon^{0} e \otimes A^{2 n+2 m+1},} \\
& {\left[h \otimes A^{2 n+1}, e \otimes A^{2 m}\right]_{\varepsilon}=\varepsilon e \otimes A^{2 n+2 m+1},} \\
& {\left[h \otimes A^{2 n+1}, e \otimes A^{2 m+1}\right]_{\varepsilon}=\varepsilon\left(e \otimes A^{2 n+2 m+2}+3 e_{1} e \otimes A^{2 n+2 m}\right.} \\
& \left.+\left(e_{1}-e_{2}\right)\left(2 e_{1}+e_{2}\right) e \otimes A^{2 n+2 m-2}\right) ; \\
& {\left[h \otimes A^{2 n}, f \otimes A^{2 m}\right]_{\varepsilon}=-\varepsilon^{0} f \otimes A^{2 n+2 m},} \\
& {\left[h \otimes A^{2 n}, f \otimes A^{2 m+1}\right]_{\varepsilon}=-\varepsilon^{0} f \otimes A^{2 n+2 m+1},} \\
& {\left[h \otimes A^{2 n+1}, f \otimes A^{2 m}\right]_{\varepsilon}=-\varepsilon f \otimes A^{2 n+2 m+1},} \\
& {\left[h \otimes A^{2 n+1}, f \otimes A^{2 m+1}\right]_{\varepsilon}=-\varepsilon\left(f \otimes A^{2 n+2 m+2}+3 e_{1} f \otimes A^{2 n+2 m}\right.} \\
& \left.+\left(e_{1}-e_{2}\right)\left(2 e_{1}+e_{2}\right) f \otimes A^{2 n+2 m-2}\right) ; \\
& {\left[e \otimes A^{2 n}, f \otimes A^{2 m}\right]_{\varepsilon}=2 \varepsilon^{0} h \otimes A^{2 n+2 m},} \\
& {\left[e \otimes A^{2 n}, f \otimes A^{2 m+1}\right]_{\varepsilon}=2 \varepsilon h \otimes A^{2 n+2 m+1},} \\
& {\left[e \otimes A^{2 n+1}, f \otimes A^{2 m}\right]_{\varepsilon}=2 \varepsilon h \otimes A^{2 n+2 m+1},}
\end{aligned}
$$




$$
\begin{aligned}
{\left[e \otimes A^{2 n+1}, f \otimes A^{2 m+1}\right]_{\varepsilon}=} & 2 \varepsilon^{0}\left(h \otimes A^{2 n+2 m+2}+3 e_{1} h \otimes A^{2 n+2 m}\right. \\
& \left.+\left(e_{1}-e_{2}\right)\left(2 e_{1}+e_{2}\right) h \otimes A^{2 n+2 m-2}\right) .
\end{aligned}
$$

In the limit $\varepsilon \rightarrow 0$, they become

$$
\begin{aligned}
& {\left[h \otimes A^{2 n}, e \otimes A^{2 m}\right]^{\prime}=e \otimes A^{2 n+2 m},} \\
& {\left[h \otimes A^{2 n}, e \otimes A^{2 m+1}\right]^{\prime}=e \otimes A^{2 n+2 m+1},} \\
& {\left[h \otimes A^{2 n+1}, e \otimes A^{2 m}\right]^{\prime}=0,} \\
& {\left[h \otimes A^{2 n+1}, e \otimes A^{2 m+1}\right]^{\prime}=0 ;} \\
& {\left[h \otimes A^{2 n}, f \otimes A^{2 m}\right]^{\prime}=-f \otimes A^{2 n+2 m},} \\
& {\left[h \otimes A^{2 n}, f \otimes A^{2 m+1}\right]^{\prime}=-f \otimes A^{2 n+2 m+1},} \\
& {\left[h \otimes A^{2 n+1}, f \otimes A^{2 m}\right]^{\prime}=0,} \\
& {\left[h \otimes A^{2 n+1}, f \otimes A^{2 m+1}\right]^{\prime}=0 ;} \\
& {\left[e \otimes A^{2 n}, f \otimes A^{2 m}\right]^{\prime}=2 h \otimes A^{2 n+2 m},} \\
& {\left[e \otimes A^{2 n}, f \otimes A^{2 m+1}\right]^{\prime}=0,} \\
& {\left[e \otimes A^{2 n+1}, f \otimes A^{2 m}\right]^{\prime}=0,} \\
& {\left[e \otimes A^{2 n+1}, f \otimes A^{2 m+1}\right]^{\prime}=2\left(h \otimes A^{2 n+2 m+2}+3 e_{1} h \otimes A^{2 n+2 m}\right.} \\
& \left.\quad+\left(e_{1}-e_{2}\right)\left(2 e_{1}+e_{2}\right) h \otimes A^{2 n+2 m-2}\right) .
\end{aligned}
$$

This contraction is interesting because it cannot be seen as the result of an affinization of a contraction of $\mathfrak{s l}_{2}(\mathbb{C})$. Indeed, it is not an affinization of $\mathfrak{r}_{3,-1}(\mathbb{C})$, in which case all the commutation relations of type $[e, f]$, in equation (19), would vanish, unlike the commutation relations above. This is similar for $\mathfrak{n}_{3}(\mathbb{C})$, for which the Lie brackets of types $[h, e]$ and $[h, f]$ (equations (17), (18) ) do vanish, unlike the Lie brackets obtained above. Also, we cannot see the contractions above as involving only the even versus odd powers of the Laurent polynomials because the grading involves also the underlying finite-dimensional Lie algebra. Indeed we find in the contracted Lie algebra that the Lie brackets are not uniquely determined by the parity of their elements; for instance, some commutators of elements both even do commute, whereas other such commutators do not commute.

Clearly, numerous other limits can be obtained, but our purpose here was just to demonstrate how a combination of deformations and contractions can lead to new Lie algebras.

\section{Acknowledgements}

Partial support was provided by a grant from NATO Hungary. AF is grateful to OTKA (Hungary) for grants T043641 and T043034. MdM acknowledges partial financial support from the Natural Sciences and Engineering Research Council of Canada, and the organizers of the Conference Symmetry in Nonlinear Mathematical Physics, held in Kyiv in June 2005. We thank both referees for their remarks and useful suggestions.

[1] Fialowski A., de Montigny M., Contractions and deformations of Lie algebras, J. Phys. A: Math. Gen., 2005, V.38, 6335-6349.

[2] Gerstenhaber M., On the deformation of rings and algebras, Ann. Math., 1964, V.79, 59-103; Gerstenhaber M., On the deformation of rings and algebras II, Ann. Math., 1966, V.84, 1-19; Gerstenhaber M., On the deformation of rings and algebras III, Ann. Math., 1968, V.88, 1-34; Gerstenhaber M., On the deformation of rings and algebras IV, 1974, V.99, 257-276; Nijenhuis A., Richardson R.W., Deformations of Lie algebra structures, J. Math. Mech., 1967, V.17, 89-105; Fialowski A., Deformations of Lie algebras, Math. USSR Sbornik, 1986, V.55, 467-472;

Fialowski A., An example of formal deformations of Lie algebras, in Proceedings of NATO Conference on 
Deformation Theory of Algebras and Applications, Editors M. Hazawinkel and M. Gerstenhaber, Dordrecht, Kluwer, 1988, 375-401;

Fialowski A., Fuchs D., Construction of miniversal deformations of Lie algebras, J. Funct. Anal., 1999, V.161, 76-110, math.RT/0006117.

[3] Fialowski A., Schlichenmaier M., Global deformations of the Witt algebra of Krichever-Novikov type, Commun. Contemp. Math., 2003, V.5, 921-945, math.QA/0206114;

Fialowski A., Schlichenmaier M., Global geometric deformations of current algebras as Krichever-Novikov type algebras, Comm. Math. Phys., 2005, V.260, 579-612, math.QA/0412113.

[4] Inönü E., Wigner E.P., On the contraction of groups and their representations, Proc. Nat. Acad. Sci. U.S.A., 1953, V.39, 510-524;

Saletan E., Contraction of Lie groups, J. Math. Phys., 1961, V.2, 1-21;

Gilmore R., Lie groups, Lie algebras, and some of their applications, New York, Wiley, 1974, Chapter 10;

Talman J.D., Special functions: a group theoretic approach, New York, Benjamin, 1968.

[5] Onishchik A.L., Vinberg E.B., Lie groups and Lie algebras, Enclycopaedia of Mathematical Sciences, Vol. 41, Berlin, Springer, 1991, Chapter 7.

[6] Weimar-Woods E., Contractions, generalized Inönü-Wigner contractions and deformations of finite-dimensional Lie algebras, Rev. Math. Phys., 2000, V.12, 1505-1529;

Lévy-Nahas M., Deformation and contraction of Lie algebras, J. Math. Phys., 1967, V.8, 1211-1222;

Lõhmus J., Tammelo R., Contractions and deformations of space-time algebras I. General theory and kinematical algebras, Hadronic J., 1997, V.20, 361-416;

Fialowski A., O'Halloran J., A comparison of deformations and orbit closure, Comm. Algebra, 1990, V.18, 4121-4140.

[7] Conatser C.W., Contractions of the low-dimensional real Lie algebras, J. Math. Phys., 1972, V.13, $196-203$.

[8] Burde D., Steinhoff C., Classification of orbit closures of 4-dimensional complex Lie algebras, J. Algebra, 1999, V.214, 729-739.

[9] Fialowski A., Deformations of some infinite-dimensional Lie algebras, J. Math. Phys., 1990, V.31, 1340-1343.

[10] Lecomte P.B.A., Roger C., Rigidity of current Lie algebras of complex simple type, J. London Math. Soc. (2), 1988, V.37, 232-240.

[11] Fialowski A., Penkava M., Versal deformations of three-dimensional Lie algebras as $L_{\infty}$ algebras, Commun. Contemp. Math., 2005, V.7, 145-165, math.RT/0303346.

[12] Goddard P., Olive D., Kac-Moody and Virasoro algebras, New York, World Scientific, 1988; Di Francesco P., Mathieu P., Sénéchal D., Conformal field theory, New York, Springer, 1997; Tsvelik A.M., Quantum field theory in condensed matter physics, Cambridge Univ. Press, 2003.

[13] Krichever I.M., Novikov S.P., Algebras of Virasoro type, Riemann surfaces and structures of the theory of solitons, Funct. Anal. Appl., 1987, V.21, 126-142;

Krichever I.M., Novikov S.P., Virasoro-type algebras, Riemann surfaces and strings in Minkowski space, Funct. Anal. Appl., 1987, V.21, 294-307;

Krichever I.M., Novikov S.P., Algebras of Virasoro type, energy-momentum tensor and decomposition operators on Riemann surfaces, Funct. Anal. Appl., 1989, V.23, 19-33.

[14] Majumdar P., Inönü-Wigner contraction of Kac-Moody algebras, J. Math. Phys., 1993, V.34, 2059-2065, hep-th/9207057;

Olive D.I., Rabinovici E., Schwimmer A., A class of string backgrounds as a semiclassical limit of WZW models, Phys. Lett. B, 1994, V.321, 361-364, hep-th/9311081. 